\title{
Pharmacist's Knowledge of Forensic Pharmacy Services
}

\author{
Yousef Ahmed Alomi*, (D) BSC. \\ Pharm, MSc. Clin Pharm, BCPS, BCNSP, DiBA, \\ CDE \\ Critical Care Clinical Pharmacists, TPN Clinical \\ Pharmacist, Freelancer Business Planner, \\ Content Editor, and Data Analyst, Riyadh, \\ SAUDI ARABIA \\ Rehab Sultan Najmi, Bsc. Pharm, \\ King Khalid University Abha, SAUDI ARABIA. \\ Mawadah Mohsen Aqeeli, \\ Pharm D, Ministery of Heath, Abha, \\ SAUDI ARABIA. \\ Samiyah Ibrahim Qassadi, \\ Pharm D, Jazan University, Jazan, \\ SAUDI ARABIA.

\section{Correspondence:} \\ Dr. Yousef Ahmed Alomi, Bsc. Pharm, Msc. \\ Clin pharm, BCPS, BCNSP, DiBA, CDE Critical \\ Care Clinical Pharmacists, TPN Clinical Phar- \\ macist, Freelancer Business Planner, Content \\ Editor and Data Analyst, P.O.BOX 100, Riyadh \\ 11392, Riyadh, SAUDI ARABIA
}

Phone no: +966504417712

E-mail:yalomi@gmail.com
Received: 10-12-2020;

Accepted: 17-02-2021;

Copyright: () the author(s), publisher and licensee International Journal of Pharmacology and Clinical Sciences. This is an open-access article distributed under the terms of the Creative Commons

Attribution Non-Commercial License, which permits unrestricted non-commercial use, distribution, and reproduction in any medium, provided the original work is properly cited.

This is an open access article distributed under the terms of the Creative Commons AttributionNonCommercial-ShareAlike 4.0 License

Access this article online

\begin{tabular}{|c|c|}
\hline & www.ijpcs.net \\
\hline & DOI: \\
\hline
\end{tabular}

\begin{abstract}
Goal: The knowledge of forensic pharmacy is a grave issue in practice. For example, forensic pharmacy services are obligatory for crime and drug-related death investigations. The current study purposes to affirm Pharmacist knowledge of forensic pharmacy in the Kingdom of Saudi Arabia. Methods: It was a qualitative examination with a cross-section design. An electronic validated and reliability survey was dispersed to all pharmacists and pharmacy interns. All students were omitted from the study. The questionnaire checked demographic data about the responders and pharmacist's basic and advanced knowledge of forensic pharmacy and resources of forensic pharmacy. Statistical Package of Social Science (SPSS), Microsoft Excel, and survey monkey did all analysis. Results: The total number of responding pharmacists was 402. Of those, $198(49.75 \%)$ were male, while $200(50.25 \%)$ were female, with statistical significance between them $(p<0.001)$. Almost three-quarters of the pharmacists had bachelor's degrees 303 (75.56\%), with statistically significant among all pharmaceutical degrees $(p<0.001)$. The total average scores of pharmacists' knowledge of forensic pharmacy services were (2.0) with high scores element was the valuation of basic knowledge of the forensic pharmacy was the medications induced addiction (2.74). In contrast, the lowest scores were the job description of forensic pharmacist (1.5). The average score advances knowledge of forensic pharmacy (1.92). The highest score knowledge was the cardiovascular medications (3.00), while the lowest score knowledge of advanced knowledge of forensic pharmacy was the resources of forensic pharmacy (1.37). The most resources applied for Forensic pharmacy services were internet $224(56.71 \%)$ and healthcare practitioners 222 (56.20\%). Conclusion: The pharmacists' knowledge of forensic pharmacy services was insufficient. Therefore, the preventing of crimes problems and drug-related death are obligatory to improve patient outcomes. The undergraduate and postgraduate education and training of forensic pharmacy services in highly recommended in Saudi Arabia.

Key words: Knowledge, Pharmacist, Forensic, Pharmacy, Saudi Arabia
\end{abstract}

\section{INTRODUCTION}

Pharmaceutical care services are increasing over the past years in Saudi Arabia or the rest of the world. ${ }^{[1-5]}$ The pharmacist shift directing from medication caring to patients cares. ${ }^{[6,7]}$ That has imitated in patient's clinical outcomes and improve the economy of the healthcare system. ${ }^{[8-12]}$ The pharmacy plays an active role model in Saudi Arabia in numerous pharmacy services. It encompassed total parenteral nutrition, anticoagulation services, drug information services, and pharmacy critical care services. ${ }^{[8-12]}$ However, some medical fields want the pharmacist to share more with treating or management teams counting nuclear medicine, forensic medicine, and other subspecialties of paediatrics. Forensic medicine is well recognised in Saudi Arabia, and there is a job by the name of the forensic pharmacist. However, most active jobs in this field are working at poisoning centres. Recently, the healthcare ministry composed all forensic medications active under one department and called forensic medicine services. It has entailed of forensic medicine, poisoning center, forensic laboratories, and related issues. As a result, the pharmacist will play an active role in the new administration. Various studies discovered the job descriptions of forensic pharmacists and forensic clinical pharmacists. ${ }^{[13,14]}$ Besides, the jobs of the forensic pharmacy technicians. They applied various activities but not limited to drug information resources of any toxic material or drug poising, the analysis inside of forensic material, investigation of drugrelated morbidity and mortality. ${ }^{[15-17]}$ Forensic pharmacists necessity the knowledge to perform their jobs to take care of new jobs of forensic pharmacy. Various publications discussed forensic pharmacy and the role of pharmacists. However, the author is not conversant with any studies about pharmacist's knowledge of forensic pharmacy in Saudi Arabia or Arabic countries, or the rest of the world. The objective of the current examination is to affirm the pharmacist's knowledge of forensic pharmacy in Saudi Arabia.

\section{METHODS}

It scrutinises a cross-sectional survey that conversed pharmacists knowledge of forensic pharmacy in Saudi Arabia. It self-reported an electronic survey of the pharmacist, counting pharmacists from internship to consultant, 
pharmacist specialties, and Saudi Arabia. All non- pharmacist or students and noncompleted surveys will be omitted from the study. The survey consisted of respondents' demographic information about pharmacists and basic and advanced knowledge of selected forensic pharmacy elements, and the resources of knowledge of forensic pharmacy elements in pharmaceutical care. The 5-point Likert response scale system was applied with closedended questions. According to the previous literature with unlimited population size, the sample was calculated as a cross-sectional study, the confidence level $95 \%$ with $\mathrm{z}$ score of 1.96 and margin of error $5 \%$, population percentage $50 \%$, and drop-out rate $10 \%$. As a result, the sample size will equal 380-420 with a power of study of $80 \% .{ }^{[18-20]}$ The response rate obligatory of calculated sample size at least $60-70 \%$ and above. ${ }^{[20,21]}$ The survey was dispersed through social media of what's applications and telegram groups of pharmacists. The reminder message had been sent every 1-2 weeks. The survey was authenticated through the revision of expert reviewers and pilot testing. Besides, numerous tests of reliability McDonald's $\omega$, Cronbach alpha, Guttmann's $\lambda 2$, and Guttmann's $\lambda 6$ had been done with the study. The data analysis of the pharmacist's knowledge of forensic pharmacy is completed through the survey monkey system. Besides, the statistical package of social sciences (SPSS), Jeffery's Amazing Statistics Program (JASP), and Microsoft excel sheet version 16. It encompassed a description and frequency analysis, good of fitness analysis, correlation analysis. Beside, inferential analysis of factors touching pharmacist's knowledge of nuclear pharmacy and radiopharmaceutical products and linear regression. The STROBE (Strengthening the reporting of observational studies in epidemiology statement: guidelines for reporting observational studies) steered the reporting of the existing study. ${ }^{[22,23]}$

\section{RESULTS}

The total number of responding pharmacists was 402 , with most of them coming from the south area $252(62.69 \%)$ with statistically noteworthy among the regions $(p<0.001)$. Of those, $198(49.75 \%)$ were male, while $200(50.25 \%)$ were female, with statistical significance between them $(p<0.001)$. Most of the responders were in age (24-30) years $269(67.08 \%)$ with statistically momentous between all ages level $(p<0.001)$. Almost threequarters of the pharmacists had bachelor's degrees 303 (75.56\%), with statistically important among all pharmaceutical degrees $(p<0.001)$. The majority of responders worked at community pharmacy 124 (30.85\%), $\mathrm{MOH}$ hospitals 107 (26.62\%), and military hospitals 76 (18.91\%). Most pharmacists were staff pharmacists 284 (70.65\%), and half of the responders had experienced three years and less 213 (53.25\%) with the majority of their practice at the community pharmacy $115(31.86 \%)$ and outpatient pharmacy 88 $(24.38 \%)$ with statistically substantial between them $(p<0.001)$. There is a strong positive association between Age (years) and Years of experience at pharmacy career Kendall's tau_b (0.699) or Spearman's rho (0.747) with statistically significant difference $(p<0.001)$. On the other hand, there is a negative medium relationship between position and age or years of experience at pharmacy career with Kendall's tau_b $(-0.447)$ or Spearman's rho $(-0.488)$ or Kendall's tau_b $(-0.460)$ or Spearman's rho (-0.511) respectively with statistically important difference $(p>0.05)$ as explored in Table 1 and 2.

The total average scores of pharmacists' knowledge of forensic pharmacy services were 2.0 with high scores element was the valuation of basic knowledge of the forensic pharmacy was the medications induced addiction (2.74), the narcotics and controlled medications law (2.67), and Medication Errors or medication safety or patient safety center (2.42). In contrast, the lowest scores were the job description of forensic pharmacist (1.5), and the forensic

\begin{tabular}{|l|c|c|c|}
\hline Table 1: Demographic information. \\
\hline Nationality & Response Count & Response Percent & p-value (X2) \\
\hline Central area & 72 & $17.91 \%$ & $<0.001$ \\
\hline North area & 24 & $5.97 \%$ & \\
\hline South area & 252 & $62.69 \%$ & \\
\hline East area & 11 & $2.74 \%$ & \\
\hline West area & 43 & $10.70 \%$ & \\
\hline Answered question & $\mathbf{4 0 2}$ & & \\
\hline Skipped question & $\mathbf{0}$ & & \\
\hline Gender & Response Count & Response Percent & \\
\hline Male & 198 & $49.75 \%$ & \\
\hline Female & 200 & $50.25 \%$ & \\
\hline Answered question & 398 & & \\
\hline Skipped question & $\mathbf{4}$ & & \\
\hline Age & Response Count & Response Percent & \\
\hline $24-30$ & 269 & $67.08 \%$ & \\
\hline $31-35$ & 73 & $18.20 \%$ & \\
\hline $36-40$ & 28 & $6.98 \%$ & \\
\hline $41-45$ & 9 & $2.24 \%$ & \\
\hline $46-50$ & $\mathbf{1}$ & $2.00 \%$ & \\
\hline$>50$ & 14 & $3.49 \%$ & \\
\hline Answered question & & & \\
\hline Skipped question & & & \\
\hline
\end{tabular}

pharmacist gets more salary than regular pharmacist (1.5) with statistically substantial between answers $(p<0.001)$ as discovered in Table 3. The average score advances knowledge of forensic pharmacy (1.92). In contrast, the highest score knowledge was the cardiovascular medications (3.00), the medication causes malformation of foetus abortion (2.78), the medications cause permanent disability (2.60), and know the anaesthetic medications (2.48). On the contrary, the lowest score knowledge of advanced knowledge of forensic pharmacy was the resources of forensic pharmacy (1.37), the clinical forensic pharmacist (1.38), and the international guidelines of forensic pharmacy (1.40), with the statistically noteworthy difference among the answers $(p<0.001)$ as explored in Table 4 . The most resources used for forensic pharmacy services were internet 224 (56.71\%), Healthcare practitioners 222 (56.20\%), Drug information resources 190 (48.10\%), and SFDA website 189 (47.85\%), as explored in Table 5. The reliability test of Guttmann's $\lambda 2,0.978$, and Guttmann's $\lambda 60.987$.

Factors persuading the forensic pharmacy basic knowledge and forensic pharmacy assessment of advance knowledge.

The numerous factors might impact the forensic pharmacy's basic knowledge. Using McDonald's $\omega, 0.978$, Cronbach alpha 0.977, 


\begin{tabular}{|c|c|c|c|}
\hline $\begin{array}{l}\text { Pharmacist } \\
\text { Qualifications }\end{array}$ & $\begin{array}{l}\text { Response } \\
\text { Count }\end{array}$ & $\begin{array}{c}\text { Response } \\
\text { Percent }\end{array}$ & $\begin{array}{l}\text { p-value } \\
\text { (X2) }\end{array}$ \\
\hline MOH Hospitals & 107 & $26.62 \%$ & $<001$ \\
\hline Military hospitals & 76 & $18.91 \%$ & \\
\hline National Gaurd Hospital & 11 & $2.74 \%$ & \\
\hline Security forces hospitals & 4 & $1.00 \%$ & \\
\hline University hospital & 12 & $2.99 \%$ & \\
\hline $\begin{array}{l}\text { MOH primary care } \\
\text { centers }\end{array}$ & 15 & $3.73 \%$ & \\
\hline Private hospitals & 12 & $2.99 \%$ & \\
\hline $\begin{array}{l}\text { Private ambulatory care } \\
\text { clinics }\end{array}$ & 1 & $0.25 \%$ & \\
\hline $\begin{array}{l}\text { Private primary healthcare } \\
\text { center }\end{array}$ & 6 & $1.49 \%$ & \\
\hline Community pharmacy & 124 & $30.85 \%$ & \\
\hline Pharmaceutical company & 15 & $3.73 \%$ & \\
\hline University (Academia) & 9 & $2.24 \%$ & \\
\hline Retried & 2 & $0.50 \%$ & \\
\hline Un-employment & 8 & $1.99 \%$ & \\
\hline Answered question & 402 & & \\
\hline Skipped question & $\mathbf{0}$ & & \\
\hline Academic Qualifications & $\begin{array}{l}\text { Response } \\
\text { Count }\end{array}$ & $\begin{array}{c}\text { Response } \\
\text { Percent }\end{array}$ & \\
\hline Diploma in Pharmacy & 29 & $7.23 \%$ & \\
\hline Bachelor's in pharmacy & 303 & $75.56 \%$ & \\
\hline Master & 47 & $11.72 \%$ & \\
\hline Pharm D & 73 & $18.20 \%$ & \\
\hline Ph. D & 9 & $2.24 \%$ & \\
\hline PGY 1 & 6 & $1.50 \%$ & \\
\hline PGY 2 & 8 & $2.00 \%$ & \\
\hline PGY 3 & 4 & $1.00 \%$ & \\
\hline Fellowship & 1 & $0.25 \%$ & \\
\hline Other (please specify) & 0 & $0.00 \%$ & \\
\hline Answered question & 401 & & \\
\hline Skipped question & 1 & & \\
\hline Position Held & $\begin{array}{l}\text { Response } \\
\text { Count }\end{array}$ & $\begin{array}{c}\text { Response } \\
\text { Percent }\end{array}$ & \\
\hline Director of Pharmacy & 18 & $4.48 \%$ & $<0.001$ \\
\hline $\begin{array}{l}\text { Assistant Director of } \\
\text { Pharmacy }\end{array}$ & 18 & $4.48 \%$ & \\
\hline Supervisor & 32 & $7.96 \%$ & \\
\hline Pharmacy staff & 284 & $70.65 \%$ & \\
\hline Pharmacy Intern & 50 & $12.44 \%$ & \\
\hline Answered question & 402 & & \\
\hline Skipped question & 0 & & \\
\hline
\end{tabular}

continued...

\begin{tabular}{|c|c|c|c|}
\hline $\begin{array}{l}\text { Years of experience at } \\
\text { Physician career }\end{array}$ & $\begin{array}{l}\text { Response } \\
\text { Count }\end{array}$ & $\begin{array}{l}\text { Response } \\
\text { Percent }\end{array}$ & \\
\hline Less than one year & 213 & $53.25 \%$ & \multirow[t]{6}{*}{$<001$} \\
\hline $1-3$ & 87 & $21.75 \%$ & \\
\hline $4-6$ & 41 & $10.25 \%$ & \\
\hline $7-9$ & 15 & $3.75 \%$ & \\
\hline $10-12$ & 12 & $3.00 \%$ & \\
\hline$>12$ & 32 & $8.00 \%$ & \\
\hline Answered question & 400 & & \\
\hline Skipped question & 2 & & \\
\hline The practice area & $\begin{array}{l}\text { Response } \\
\text { Count }\end{array}$ & $\begin{array}{c}\text { Response } \\
\text { Percent }\end{array}$ & \\
\hline Inpatient Pharmacy & 46 & $12.74 \%$ & \multirow[t]{18}{*}{$<001$} \\
\hline Outpatient Pharmacy & 88 & $24.38 \%$ & \\
\hline Satellite Pharmacy & 3 & $0.83 \%$ & \\
\hline Narcotics and Controlled & 4 & $1.11 \%$ & \\
\hline $\begin{array}{l}\text { Extemporaneous } \\
\text { Preparation }\end{array}$ & 1 & $0.28 \%$ & \\
\hline Clinical Pharmacy & 46 & $12.74 \%$ & \\
\hline Inventory Control & 8 & $2.22 \%$ & \\
\hline Drug Information & 5 & $1.39 \%$ & \\
\hline IV admixture & 11 & $3.05 \%$ & \\
\hline Pharmacy informatics & 1 & $0.28 \%$ & \\
\hline $\begin{array}{l}\text { Hospital Pharmacy } \\
\text { administration }\end{array}$ & 4 & $1.11 \%$ & \\
\hline Forensic medicine & 1 & $0.28 \%$ & \\
\hline Community pharmacy & 115 & $31.86 \%$ & \\
\hline Lecturer (Academia) & 2 & $0.55 \%$ & \\
\hline Pharmaceutical company & 14 & $3.88 \%$ & \\
\hline Non-specific & 12 & $3.32 \%$ & \\
\hline Answered question & 361 & & \\
\hline Skipped question & 41 & & \\
\hline
\end{tabular}

independent samples KruskalWallis test and the Bonferroni correction for multiple tests have adjusted significant values, the results showed as follows. Five locations exaggerated the knowledge, with south the lowest average score (1.5671) with statically significant differences $\quad(p=0.000)$. Thirteen levels of the work site pretentious the knowledge that community pharmacy had the lowest average score (1.6749) with statistically significant differences $(p=0.000)$. Six groups of age affected the knowledge with age (24-30 years) lowest average score (1.8038) with statically noteworthy differences $(p=0.000)$. There are statically significant differences in the inducing the forensic pharmacy basic knowledge in gender ( $p=0.000)$, stressing male more basic knowledge than the female of the average score (2.1536). Five levels of a position affected the knowledge with pharmacy intern's lowest average score (1.2701) with statically weighty differences $(p=0.000)$. Finally, six groups of experiences convoluted the knowledge with less than one year of background with the lowest average score (1.7106) with statistically important differences $(p=0.000)$. 


\begin{tabular}{|c|c|c|c|c|c|c|c|c|c|c|c|c|c|}
\hline \multirow{2}{*}{$\begin{array}{l}\text { Have you ever heard about } \\
\text { the concept of forensic } \\
\text { pharmacy? }\end{array}$} & \multicolumn{2}{|c|}{$\begin{array}{l}\text { There is no } \\
\text { Knowledge }\end{array}$} & \multicolumn{2}{|c|}{$\begin{array}{c}1-25 \% \\
\text { Knowledge }\end{array}$} & \multicolumn{2}{|c|}{$\begin{array}{c}26-50 \% \\
\text { Knowledge }\end{array}$} & \multicolumn{2}{|c|}{$\begin{array}{c}51-75 \% \\
\text { Knowledge }\end{array}$} & \multicolumn{2}{|c|}{$\begin{array}{c}76-100 \\
\text { Knowledge }\end{array}$} & \multirow{2}{*}{$\begin{array}{c}\text { Total } \\
\\
402\end{array}$} & \multirow{2}{*}{$\begin{array}{c}\text { Weighted } \\
\text { Average } \\
1.65\end{array}$} & \multirow{2}{*}{$\begin{array}{c}\begin{array}{c}p \text {-value } \\
(\text { X2) }\end{array} \\
<001\end{array}$} \\
\hline & $65.17 \%$ & 262 & $16.92 \%$ & 68 & $7.96 \%$ & 32 & $7.46 \%$ & 30 & $2.49 \%$ & 10 & & & \\
\hline $\begin{array}{l}\text { Have you ever heard about } \\
\text { the concept of a forensic } \\
\text { pharmacist job? }\end{array}$ & $66.17 \%$ & 266 & $16.92 \%$ & 68 & $8.21 \%$ & 33 & $5.72 \%$ & 23 & $2.99 \%$ & 12 & 402 & 1.62 & $<001$ \\
\hline $\begin{array}{l}\text { In Saudi Arabia, are there } \\
\text { legal provisions in the } \\
\text { medicines act that provide } \\
\text { for Medication Errors (MEs) } \\
\text { activities? }\end{array}$ & $33.58 \%$ & 135 & $30.85 \%$ & 124 & $14.18 \%$ & 57 & $12.69 \%$ & 51 & $8.71 \%$ & 35 & 402 & 2.32 & $<001$ \\
\hline $\begin{array}{l}\text { In Saudi Arabia, is there an } \\
\text { official crime or problem? }\end{array}$ & $48.13 \%$ & 193 & $20.45 \%$ & 82 & $11.47 \%$ & 46 & $6.98 \%$ & 28 & $12.97 \%$ & 52 & 401 & 2.16 & $<001$ \\
\hline $\begin{array}{l}\text { Do you know the severity } \\
\text { classification for Medication } \\
\text { Errors (MEs)? }\end{array}$ & $52.24 \%$ & 210 & $16.92 \%$ & 68 & $10.95 \%$ & 44 & $8.46 \%$ & 34 & $11.44 \%$ & 46 & 402 & 2.1 & $<001$ \\
\hline $\begin{array}{l}\text { Do you know the system of } \\
\text { medications errors sentinel } \\
\text { events? }\end{array}$ & $70.00 \%$ & 280 & $9.75 \%$ & 39 & $10.00 \%$ & 40 & $5.00 \%$ & 20 & $5.25 \%$ & 21 & 400 & 1.66 & $<001$ \\
\hline $\begin{array}{l}\text { Do you know the narcotics } \\
\text { and controlled medications } \\
\text { law? }\end{array}$ & $18.66 \%$ & 75 & $39.05 \%$ & 157 & $15.42 \%$ & 62 & $10.20 \%$ & 41 & $16.67 \%$ & 67 & 402 & 2.67 & $<001$ \\
\hline $\begin{array}{l}\text { Do you know the medical } \\
\text { discrimination system? }\end{array}$ & $56.97 \%$ & 229 & $17.41 \%$ & 70 & $11.69 \%$ & 47 & $7.96 \%$ & 32 & $5.97 \%$ & 24 & 402 & 1.89 & $<001$ \\
\hline $\begin{array}{l}\text { In Saudi Arabia, the forensic } \\
\text { pharmacist get more salary } \\
\text { than regular pharmacist }\end{array}$ & $74.81 \%$ & 300 & $12.22 \%$ & 49 & $4.74 \%$ & 19 & $4.49 \%$ & 18 & $3.74 \%$ & 15 & 401 & 1.5 & $<001$ \\
\hline Answered & & & & & & & & & & & & 402 & \\
\hline Skipped & & & & & & & & & & & & 0 & \\
\hline
\end{tabular}

The numerous factor might influence the forensic pharmacy valuation of advanced knowledge. Five locations exaggerated the knowledge, with south the lowest average score (1.5669) with statically significant differences $(p=0.000)$. Thirteen levels of the work site affected the knowledge that community pharmacy had the lowest average score (1.6923) with statistically important differences $(p=0.000)$. Six groups of age exaggerated the knowledge with age (24-30 years) lowest average score (1.7875) with statically significant differences $(p=0.000)$. Regarding gender, the male has more knowledge than the female of the average score (2.0449) with statically significant differences in the manipulating the Forensic pharmacy advance knowledge $(p=0.007)$. Five levels of a position affected the knowledge with pharmacy intern's lowest average score (1.3431) with statically significant differences $(p=0.000)$. Finally, six groups of the level of experiences affected the knowledge with less than one year experiences had a lowest average score (1.7198) with statically significant differences $(p=0.000)$ as explored in Table 6.
The relationship between forensic pharmacy basic knowledge and factors location, site of work, age (years), pharmacist gender, years of experience in pharmacy career, and position Held. It was revealed through a multiple regression model and measured the Forensic pharmacy basic knowledge dependent variable, and factors were measured an expletory variable. There was a medium relationship R (0.451) with ( $\mathrm{p}=0.000)$ between Forensic pharmacy basic knowledge and factors. All factors were non-significant differences $(p>0.05)$. However, there are only 
Table 4: Forensic pharmacy assessment of advanced knowledge.

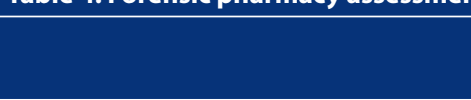

Do you know the analysis of forensic medications ions?

Do you know medications induced death

Do you know the medications cause permanent disability?

Do you know the Medication causes malformation of fetus abortion?

Do you know the narcotics or controlled medications during a medical examination for a new job?

Do you know about toxic-kinetics used in forensic pharmacy?

Do you know the cosmetics used in forensic pharmacy?

Do you know the international guidelines of forensic pharmacy?

Do you know the clinical forensic pharmacist?

Do you know the off-labeled or nonapproved medications?

Do you know the anesthetic medications?

Do you know the cardiovascular medications?

Do you know the factors affecting hiding results during drug analysis?

Do you now herbal contributed permanent disabilities or death

Do you know forensic toxicology

Do you know the resources of forensic pharmacy

\section{Answered}

Skipped

\begin{tabular}{|c|c|c|c|c|c|c|c|c|c|c|c|c|}
\hline \multicolumn{2}{|c|}{$\begin{array}{l}\text { There is no } \\
\text { Knowledge }\end{array}$} & \multicolumn{2}{|c|}{$\begin{array}{c}1-25 \% \\
\text { Knowledge }\end{array}$} & \multicolumn{2}{|c|}{$\begin{array}{c}26-50 \% \\
\text { Knowledge }\end{array}$} & \multicolumn{2}{|c|}{$\begin{array}{c}51-75 \% \\
\text { Knowledge }\end{array}$} & \multicolumn{2}{|c|}{$\begin{array}{c}76-100 \\
\text { Knowledge }\end{array}$} & \multirow{2}{*}{$\begin{array}{c}\text { Total } \\
401\end{array}$} & \multirow{2}{*}{$\begin{array}{c}\begin{array}{c}\text { Weighted } \\
\text { Average }\end{array} \\
1.56\end{array}$} & \multirow{2}{*}{$\begin{array}{c}\begin{array}{c}\boldsymbol{p} \text {-value } \\
(\mathbf{X})\end{array} \\
<001\end{array}$} \\
\hline $72.32 \%$ & 290 & $11.47 \%$ & 46 & $6.98 \%$ & 28 & $5.99 \%$ & 24 & $3.24 \%$ & 13 & & & \\
\hline $19.40 \%$ & 78 & $42.79 \%$ & 172 & $17.91 \%$ & 72 & $12.44 \%$ & 50 & $7.46 \%$ & 30 & 402 & 2.46 & $<001$ \\
\hline $8.25 \%$ & 33 & $50.25 \%$ & 201 & $22.00 \%$ & 88 & $12.25 \%$ & 49 & $7.25 \%$ & 29 & 400 & 2.60 & $<001$ \\
\hline $9.98 \%$ & 40 & $41.40 \%$ & 166 & $22.94 \%$ & 92 & $12.47 \%$ & 50 & $13.22 \%$ & 53 & 401 & 2.78 & $<001$ \\
\hline $46.77 \%$ & 188 & $22.64 \%$ & 91 & $10.45 \%$ & 42 & $8.71 \%$ & 35 & $11.44 \%$ & 46 & 402 & 2.15 & $<001$ \\
\hline $69.58 \%$ & 279 & $10.22 \%$ & 41 & $8.98 \%$ & 36 & $6.48 \%$ & 26 & $4.74 \%$ & 19 & 401 & 1.67 & $<001$ \\
\hline $78.11 \%$ & 314 & $8.96 \%$ & 36 & $7.46 \%$ & 30 & $2.74 \%$ & 11 & $2.74 \%$ & 11 & 402 & 1.43 & $<001$ \\
\hline $80.35 \%$ & 323 & $7.71 \%$ & 31 & $6.47 \%$ & 26 & $2.99 \%$ & 12 & $2.49 \%$ & 10 & 402 & 1.40 & $<001$ \\
\hline $80.80 \%$ & 324 & $8.48 \%$ & 34 & $4.74 \%$ & 19 & $4.24 \%$ & 17 & $1.75 \%$ & 7 & 401 & 1.38 & $<001$ \\
\hline $62.44 \%$ & 251 & $10.20 \%$ & 41 & $9.45 \%$ & 38 & $7.96 \%$ & 32 & $9.95 \%$ & 40 & 402 & 1.93 & $<001$ \\
\hline $26.62 \%$ & 107 & $34.08 \%$ & 137 & $16.42 \%$ & 66 & $10.95 \%$ & 44 & $11.94 \%$ & 48 & 402 & 2.48 & $<001$ \\
\hline $6.97 \%$ & 28 & $39.55 \%$ & 159 & $18.66 \%$ & 75 & $15.92 \%$ & 64 & $18.91 \%$ & 76 & 402 & 3.00 & $<001$ \\
\hline $75.87 \%$ & 305 & $11.19 \%$ & 45 & $5.97 \%$ & 24 & $3.23 \%$ & 13 & $3.73 \%$ & 15 & 402 & 1.48 & $<001$ \\
\hline $67.58 \%$ & 271 & $16.71 \%$ & 67 & $7.48 \%$ & 30 & $2.24 \%$ & 9 & $5.99 \%$ & 24 & 401 & 1.62 & $<001$ \\
\hline $79.95 \%$ & 319 & $7.27 \%$ & 29 & $5.01 \%$ & 20 & $5.26 \%$ & 21 & $2.51 \%$ & 10 & 399 & 1.43 & $<001$ \\
\hline \multirow[t]{3}{*}{$79.30 \%$} & 318 & $9.73 \%$ & 39 & $7.23 \%$ & 29 & $1.75 \%$ & 7 & $2.00 \%$ & 8 & 401 & 1.37 & $<001$ \\
\hline & & & & & & & & & & & 402 & \\
\hline & & & & & & & & & & & 0 & \\
\hline
\end{tabular}

Table 5: The most resources used for nuclear pharmacy information.

\section{Answer Choices}

Healthcare practitioners

Scientific literature

Peer discussions

Medical association literature/guidelines/recommendations

Drug information resources (Lexicomp-drug information, Micromedex, Epocrates ..etc

SFDA website

Drug Bulletin

Relatives and friends

Nuclear pharmacy education courses

Internet

The drug information center at the hospital

Awareness lectures in a hospital

Awareness lectures primary healthcare center

Healthcare care awareness events at the market

Answered

Skipped

\section{Responses}

\begin{tabular}{|c|c|}
\hline 222 & $56.20 \%$ \\
\hline 144 & $36.46 \%$ \\
\hline 36 & $9.11 \%$ \\
\hline 190 & $9.62 \%$ \\
\hline 189 & $48.10 \%$ \\
\hline 46 & $47.85 \%$ \\
\hline 119 & $11.65 \%$ \\
\hline 97 & $30.13 \%$ \\
\hline 224 & $24.56 \%$ \\
\hline 143 & $56.71 \%$ \\
\hline 14 & $36.20 \%$ \\
\hline 12 & $5.06 \%$ \\
\hline 395 & $3.54 \%$ \\
\hline 7 & $3.04 \%$ \\
\hline
\end{tabular}




\begin{tabular}{|c|c|c|c|c|c|c|c|c|c|c|c|c|c|c|c|}
\hline & \multirow[b]{2}{*}{ Factors } & \multicolumn{7}{|c|}{ Forensic pharmacy assessment of basic knowledge } & \multicolumn{7}{|c|}{ Forensic pharmacy assessment of advanced knowledge } \\
\hline & & $\mathbf{N}$ & $\begin{array}{l}\text { Average } \\
\text { scores }\end{array}$ & Std. D & Median & $\begin{array}{l}\text { Lower } \\
\text { Bound }\end{array}$ & $\begin{array}{l}\text { Upper } \\
\text { Bound }\end{array}$ & $P$-value & $\mathbf{N}$ & $\begin{array}{l}\text { Average } \\
\text { scores }\end{array}$ & Std. D & Median & $\begin{array}{l}\text { Lower } \\
\text { Bound }\end{array}$ & $\begin{array}{l}\text { Upper } \\
\text { Bound }\end{array}$ & P-value \\
\hline \multirow{6}{*}{$\begin{array}{l}\overline{0} \\
60 \\
\mathscr{0} \\
\widetilde{\simeq}\end{array}$} & Central & 72 & 2.8165 & .97160 & 2.7596 & 2.5882 & 3.0448 & \multirow{5}{*}{0.000} & 72 & 2.6675 & 1.00349 & 2.4063 & 2.4317 & 2.9033 & \multirow{5}{*}{0.000} \\
\hline & North & 23 & 2.5518 & .92907 & 2.6154 & 2.1501 & 2.9536 & & 23 & 2.5772 & .86495 & 2.5000 & 2.2031 & 2.9512 & \\
\hline & South & 247 & $1.5671^{\star}$ & .67464 & 1.3077 & 1.4826 & 1.6517 & & 247 & $1.5669^{*}$ & .63059 & 1.3125 & 1.4878 & 1.6459 & \\
\hline & East & 10 & 3.1462 & .82805 & 3.0769 & 2.5538 & 3.7385 & & 10 & 2.6688 & .79825 & 2.7500 & 2.0977 & 3.2398 & \\
\hline & West & 43 & 2.6133 & 1.09804 & 2.3846 & 2.2754 & 2.9512 & & 43 & 2.2295 & .97487 & 1.8750 & 1.9294 & 2.5295 & \\
\hline & Total & 395 & & & & & & & 395 & & & & & & \\
\hline \multirow{14}{*}{ 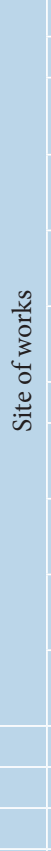 } & MOH Hospitals & 106 & 2.2092 & 1.02754 & 2.0385 & 2.0113 & 2.4071 & \multirow{13}{*}{0.000} & 106 & 2.0302 & .97580 & 1.6458 & 1.8423 & 2.2181 & \multirow{13}{*}{0.000} \\
\hline & Military hospitals & 74 & 1.6939 & .75354 & 1.3077 & 1.5193 & 1.8684 & & 74 & 1.7226 & 68951 & 1.3750 & 1.5628 & 1.8823 & \\
\hline & $\begin{array}{l}\text { National Guard } \\
\text { Hospital }\end{array}$ & 10 & 2.7231 & 1.01352 & 2.5000 & 1.9980 & 3.4481 & & 10 & 2.3063 & .43844 & 2.2500 & 1.9926 & 2.6199 & \\
\hline & $\begin{array}{l}\text { Security forces } \\
\text { hospitals }\end{array}$ & 4 & 3.0962 & .60691 & 3.0000 & 2.1304 & 4.0619 & & 4 & 3.0406 & .63273 & 3.1563 & 2.0338 & 4.0474 & \\
\hline & $\begin{array}{l}\text { University } \\
\text { hospital }\end{array}$ & 12 & 2.9808 & .91554 & 2.8846 & 2.3991 & 3.5625 & & 12 & 2.7111 & .78662 & 3.0625 & 2.2113 & 3.2109 & \\
\hline & $\begin{array}{l}\mathrm{MOH} \text { primary } \\
\text { care centers }\end{array}$ & 15 & 1.7846 & .73965 & 1.5385 & 1.3750 & 2.1942 & & 15 & 1.8056 & .76192 & 1.4375 & 1.3836 & 2.2275 & \\
\hline & Private hospitals & 11 & 2.0629 & .80591 & 1.6923 & 1.5215 & 2.6044 & & 11 & 1.8977 & .44483 & 1.7500 & 1.5989 & 2.1966 & \\
\hline & $\begin{array}{l}\text { Private primary } \\
\text { healthcare center }\end{array}$ & 6 & 1.9359 & .49713 & 1.9231 & 1.4142 & 2.4576 & & 6 & 1.8854 & 43376 & 1.9688 & 1.4302 & 2.3406 & \\
\hline & $\begin{array}{l}\text { Community } \\
\text { pharmacy }\end{array}$ & 122 & $1.6749^{*}$ & .95257 & 1.3077 & 1.5042 & 1.8457 & & 122 & $1.6923^{*}$ & .91622 & 1.3125 & 1.5281 & 1.8565 & \\
\hline & $\begin{array}{l}\text { Pharmaceutical } \\
\text { company }\end{array}$ & 15 & 2.5231 & 1.00892 & 2.2308 & 1.9644 & 3.0818 & & 15 & $2.2369^{*}$ & .99151 & 1.8125 & 1.6879 & 2.7860 & \\
\hline & $\begin{array}{l}\text { University } \\
\text { (Academia) }\end{array}$ & 9 & 2.4188 & .69243 & 2.2308 & 1.8866 & 2.9510 & & 9 & 2.2639 & 1.01251 & 1.8750 & 1.4856 & 3.0422 & \\
\hline & Retried & 2 & 2.6154 & .43514 & 2.6154 & $-1.2942-$ & 6.5250 & & 2 & 2.9063 & .92808 & 2.9063 & $-5.4322-$ & 11.2447 & \\
\hline & Un-employment & 8 & 2.9231 & .96865 & 3.0000 & 2.1133 & 3.7329 & & 8 & 2.7500 & .95080 & 2.7813 & 1.9551 & 3.5449 & \\
\hline & Total & 395 & & & & & & & 395 & & & & & & \\
\hline \multirow{7}{*}{$\stackrel{8}{\&}$} & $24-30$ & 267 & $1.8038^{*}$ & .97315 & 1.3077 & 1.6866 & 1.9211 & \multirow{6}{*}{0.000} & 267 & $1.7875^{*}$ & .92476 & 1.3750 & 1.6761 & 1.8990 & \multirow{6}{*}{0.000} \\
\hline & $31-35$ & 72 & 2.1074 & .75829 & 1.8462 & 1.9292 & 2.2856 & & 72 & 1.9571 & .64300 & 1.7500 & 1.8060 & 2.1082 & \\
\hline & $36-40$ & 26 & 2.7189 & .84320 & 2.6538 & 2.3784 & 3.0595 & & 26 & 2.5455 & .73804 & 2.3563 & 2.2474 & 2.8436 & \\
\hline & $41-45$ & 9 & 2.4615 & 1.01687 & 2.1538 & 1.6799 & 3.2432 & & 9 & 2.3958 & .93070 & 2.1875 & 1.6804 & 3.1112 & \\
\hline & $46-50$ & 7 & 3.1648 & 1.00632 & 2.9231 & 2.2341 & 4.0955 & & 7 & 2.8125 & 1.24896 & 2.7500 & 1.6574 & 3.9676 & \\
\hline & $>50$ & 14 & 3.1461 & .68960 & 2.8846 & 2.7479 & 3.5442 & & 14 & 2.5208 & .77254 & 2.2813 & 2.0748 & 2.9669 & \\
\hline & Total & 395 & & & & & & & 395 & & & & & & \\
\hline \multirow{3}{*}{$\begin{array}{l}\overrightarrow{\vec{v}} \\
\overrightarrow{0} \\
\bar{v}\end{array}$} & Male & 196 & $2.1536^{*}$ & .97880 & 1.9231 & 2.0157 & 2.2915 & \multirow{2}{*}{0.000} & 196 & $2.0449^{*}$ & .93254 & 1.6771 & 1.9136 & 2.1763 & \multirow{2}{*}{0.007} \\
\hline & Female & 199 & 1.8608 & .97981 & 1.3846 & 1.7238 & 1.9977 & & 199 & 1.8095 & .86180 & 1.3750 & 1.6891 & 1.9300 & \\
\hline & Total & 395 & & & & & & & 395 & & & & & & \\
\hline \multirow{6}{*}{ 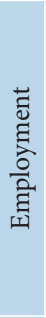 } & $\begin{array}{l}\text { Director of } \\
\text { Pharmacy }\end{array}$ & 18 & 2.4263 & .99534 & 2.6154 & 1.9313 & 2.9213 & \multirow{5}{*}{0.000} & 18 & 2.0903 & .76313 & 2.0625 & 1.7108 & 2.4698 & \multirow{5}{*}{0.000} \\
\hline & $\begin{array}{l}\text { Assistant director } \\
\text { of Pharmacy }\end{array}$ & 17 & 2.6900 & .62736 & 2.8462 & 2.3675 & 3.0126 & & 17 & 2.4853 & .70832 & 2.3125 & 2.1211 & 2.8495 & \\
\hline & Supervisor & 29 & 2.8240 & .84320 & 2.6154 & 2.5033 & 3.1448 & & 29 & 2.5507 & .82377 & 2.5000 & 2.2374 & 2.8641 & \\
\hline & Pharmacy Staff & 282 & 1.9818 & .98804 & 1.5385 & 1.8660 & 2.0976 & & 282 & 1.9193 & .92285 & 1.5000 & 1.8111 & 2.0275 & \\
\hline & Pharmacy intern & 49 & $1.2701^{\star}$ & .47364 & 1.1538 & 1.1341 & 1.4062 & & 49 & $1.3431^{\star}$ & .50164 & 1.2500 & 1.1990 & 1.4872 & \\
\hline & Total & 395 & & & & & & & 395 & & & & & & \\
\hline \multirow{7}{*}{ 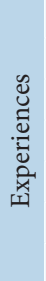 } & $<1$ & 211 & $1.7106^{*}$ & .94230 & 1.3077 & 1.5827 & 1.8384 & & 211 & $1.7198^{*}$ & .91961 & 1.3125 & 1.5950 & 1.8446 & \\
\hline & $1-3$ & 87 & 1.9446 & .87202 & 1.6154 & 1.7587 & 2.1304 & & 87 & 1.9108 & .82059 & 1.6250 & 1.7359 & 2.0857 & \\
\hline & $4-6$ & 39 & 2.4448 & .74176 & 2.3846 & 2.2043 & 2.6852 & 0000 & 39 & 2.2506 & .66328 & 2.0625 & 2.0356 & 2.4657 & 0 \\
\hline & 7-9 & 14 & 2.8077 & .77145 & 2.6538 & 2.3623 & 3.2531 & 0.000 & 14 & 2.3438 & .80329 & 2.1563 & 1.8799 & 2.8076 & .000 \\
\hline & $10-12$ & 12 & 2.7735 & .80847 & 2.6923 & 2.2598 & 3.2872 & & 12 & 2.4924 & .65119 & 2.6417 & 2.0786 & 2.9061 & \\
\hline & $>12$ & 32 & 2.9485 & .93202 & 2.8397 & 2.6125 & 3.2845 & & 32 & 2.5404 & .90449 & 2.3438 & 2.2143 & 2.8665 & \\
\hline & Total & 395 & & & & & & & 395 & & & & & & \\
\hline
\end{tabular}




\begin{tabular}{|c|c|c|c|c|c|c|c|c|c|c|c|c|c|c|}
\hline \multirow[t]{4}{*}{1} & (Constant) & $.451^{\mathrm{b}}$ & .204 & 16.530 & $.000^{\mathrm{b}}$ & 2.365 & .324 & & 7.293 & .000 & 1.727 & 3.003 & & \\
\hline & Age (years) & & & & & .027 & .065 & .033 & .422 & .674 & $-.101-$ & .156 & .332 & 3.014 \\
\hline & $\begin{array}{l}\text { Pharmacist } \\
\text { gender }\end{array}$ & & & & & $-.052-$ & .094 & $-.026-$ & $-.558-$ & .577 & $-.236-$ & .132 & .915 & 1.093 \\
\hline & $\begin{array}{l}\text { Years of } \\
\text { experience at } \\
\text { pharmacy career }\end{array}$ & & & & & .206 & .052 & .319 & 3.932 & .000 & .103 & .309 & .311 & 3.212 \\
\hline
\end{tabular}

a. Dependent Variable: Nuclear pharmacy basic knowledge, Predictors: (Constant), Location, Age (years), Pharmacist gender, Position Held, and Years of experiences at pharmacy career.

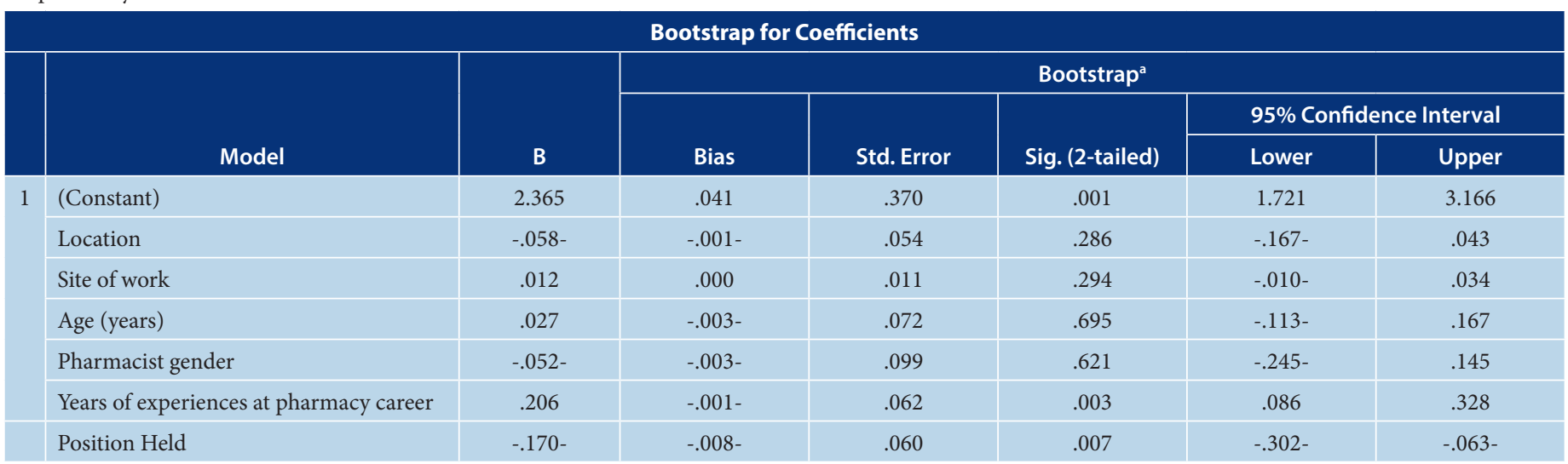

a. Unless otherwise noted, bootstrap results are based on 1000 bootstrap samples

two factors (Years of experiences) explained $31.9 \%$ positive relationship $(\mathrm{p}=0.000)$, and pharmacist's position explained $14.9 \%$ negative relationship $(p=0.004)$ the variation in the Forensic pharmacy basic knowledge through multiple regression model and confirmed by Bootstrap model. The relationship verified by the non-existence of multi-collinearity with the years of experiences factor Variance Inflation Factor (VIF=3.212) and pharmacist's position $(\mathrm{VIF}=1.279)$ almost nearest three or less than five ${ }^{[24-26]}$ as explored in Table 7 . There was a weak relationship $\mathrm{R}(0.372)$ with $(\mathrm{p}=0.000)$ between forensic pharmacy advanced knowledge and factors. All factors were non-significant differences $(p>0.05)$. However, there are only three factors locations, explained $16.3 \%$ negative relationship, (Years of experiences) explained $21.6 \%$ positive relationship $(p=0.011)$, and pharmacist's position explained $11.3 \%$ negative relationship $(p=0.035)$ the variation in the forensic pharmacy advanced knowledge through multiple regression model and confirmed by Bootstrap model.
The relationship verified by the non-existence of multi-collinearity with, locations factor Variance Inflation Factor $(\mathrm{VIF}=1.054)$, the years of experiences $(\mathrm{VIF}=3.212)$ and pharmacist's position $(\mathrm{VIF}=1.279)$ almost nearest three or less than five $\mathrm{e}^{[24-26]}$ as explored in Table 8.

\section{DISCUSSION}

There are numerous jobs for pharmacists. The pharmacist can work in the hospital pharmacy, community pharmacy, pharmaceutical companies, toxicology and forensic chemistry laboratories, and academic pharmacy, counting Universities and training centers. ${ }^{[13,27]}$ Over the past years, there were shortage of workforce in the pharmacy field. ${ }^{[28,29]}$ The ministry of education prolonged the number of colleges of pharmacies in the past years. As a result, the number of graduate pharmacists increases, and they need job vacancies. ${ }^{[30]}$ Recently, the Ministry of Human Resources lengthened the pharmacist jobs in the pharmaceutical companies and community pharmacies. In the past years, the pharmacist delivers excellent patients care services, counting clinical pharmacy specialty or medication distribution services, and is expanding by the time. ${ }^{[2,3]}$ The impact of pharmacists in drug therapy is well recognized in Saudi Arabia in several fields of pharmacy. ${ }^{[10,31-33]}$ However, the area of forensic medicine was absent the pharmacist locally or internationally. Despite, there was nominated jobs by the ministry of Human Resources. Through recent variations in the curriculum of the college of pharmacy, the pharmacist had many knowledge and practice experiences. In forensic medicine, the pharmacist can examine the toxic or intention poising material, Monitor and prevent the medication errors and drugrelated problems that lead to intentional death, assessment any dangerous drug interaction or drug-food interaction. Besides, provide and lead the poisoning management related issues, drug or substance addiction. As a result, the pharmacist desires knowledge for forensic medicine and work in the forensic pharmacy field. ${ }^{[28,29]}$ Based on the authors' best knowledge, 


\begin{tabular}{|c|c|c|c|c|c|c|c|c|c|c|c|c|}
\hline $.372^{\mathrm{b}}$ & .138 & 10.361 & $.000^{\mathrm{b}}$ & 2.478 & .308 & & 8.034 & .000 & 1.871 & 3.084 & & \\
\hline & & & & .016 & .062 & .021 & .251 & .802 & $-.106-$ & .137 & .332 & 3.014 \\
\hline & & & & $-.054-$ & .089 & $-.030-$ & $-.610-$ & .542 & -.229 & .121 & .915 & 1.093 \\
\hline & & & & .127 & .050 & .216 & 2.554 & .011 & .029 & .225 & .311 & 3.212 \\
\hline
\end{tabular}

a. Dependent Variable: Nuclear pharmacy basic knowledge, Predictors: (Constant), Location, Age (years), Pharmacist gender, Position Held, and Years of experiences at pharmacy career.

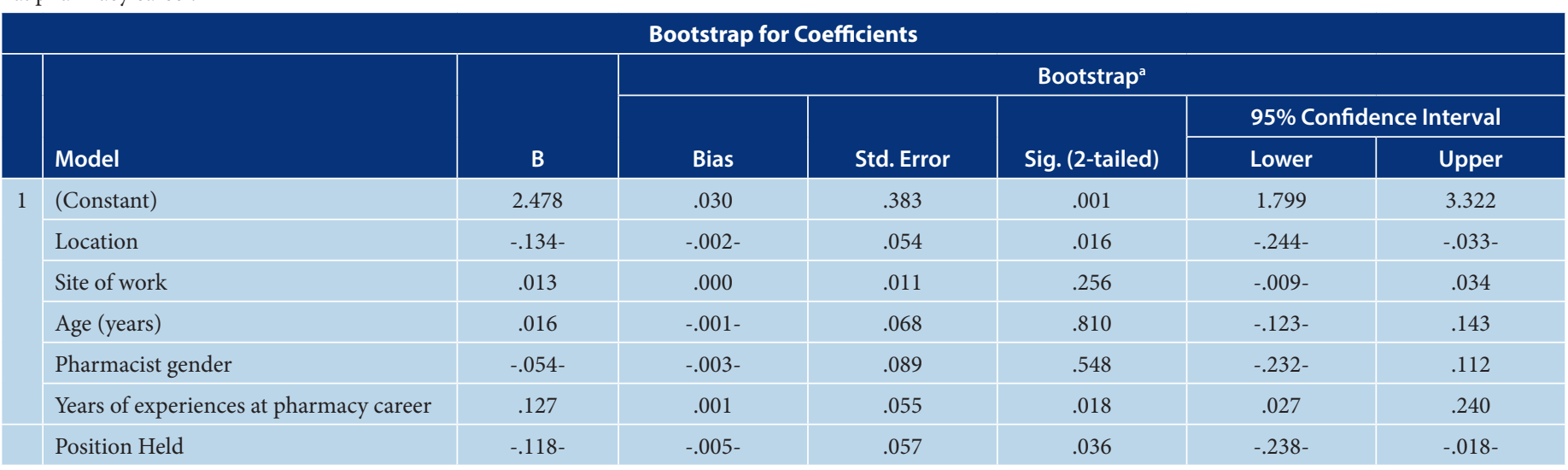

a. Unless otherwise noted, bootstrap results are based on 1000 bootstrap samples

there was little or nil investigation about pharmacist's knowledge of forensic pharmacy locally or Gulf and Middle East countries. The current study goals to declare the pharmacist knowledge of forensic pharmacy in Saudi Arabia. Based on the responder's results with suitable sample and satisfactory calculated sample size and power of eighty. Besides, it includes good validation and a high-reliability score of the various test. The majority of responders from the southern region were predictable because most of the authors were situated in the south area of Saudi Arabia and dispersed the electronic survey at their location. The gender distribution is almost the sample and resembles the Saudi population. The majority of responders were young age; which is probable because more willing to partake in the research than other groups of age. Most of the responders had a bachelor's degree, which was expected because most pharmacy colleges graduated at this level. Most of them worked at a community pharmacy, which might nonSaudi responders graduated from outside
Saudi Arabia. Those young pharmacists had low working experiences and working as community pharmacists or at the outpatient pharmacy. There is a strong positive association between age and the number of years of experience anticipated (Higher age and higher experiences). It also includes negative medium correlation employment and age that's young age with higher positions. It might be linked to younger pharmacists available for higher work in the pharmacy practice.

The average score of basic knowledge of forensic pharmacy was lacking; almost $20 \%$ only resemble of earlier forensic medicine study. ${ }^{[34]}$ The highest knowledge was about druginduced addition, the narcotics law, and the medications error system, which was expected because those elements had been executed in the pharmacy practice with close monitoring by Saudi Central Board for Accreditation of Healthcare Institutions (CBAHI). While the lowest score knowledge was the job of the forensic pharmacist or the higher salary offers to the forensic pharmacist expected from responders because most of them work at community pharmacies far away from the forensic pharmacy practice. Also, forensic pharmacy is not taught during pharmacy, poor or nil continuous education and forensic pharmacy. The hospital pharmacist did not contain the forensic pharmacy services, did not included as members of the mortality community or criminal committee. The current examination exposed the average scores of advanced knowledge of forensic pharmacy were poor. The highest knowledge was about cardiovascular medications or medications harmful to the foetus or get an abortion, significant adverse events, and anesthesia medications expected because knowledge loads daily practice and is used for forensic pharmacy knowledge.

On the contrary, the lowest advanced knowledge of forensic pharmacy was the resources of forensic pharmacy or clinical pharmacist or pharmacy international guidelines related to poor education during college of pharmacy and the bulk of responders not working in 
forensic laboratories or forensic laboratories pharmacy field. Due to poor forensic pharmacy knowledge, most responders applied healthcare practitioners or the internet, and seldom-used drug information resources look like preceding forensic medicine studies. ${ }^{[35]}$ The various current factors might be the results of essential knowledge and advanced knowledge of forensic pharmacy. It includes location, site of work, age, gender, employment, number of years of experiences with a statistically significant difference. The south region had a lower score of basic and advanced knowledge of forensic pharmacy. The reason is unclear and might be that a pharmacist does not distinguish the forensic medicine services, or the responders are not trained at forensic pharmacy services. The community pharmacist had a lower score of knowledge because it is not their practice field or not educated about forensic pharmacy. The lower age had lower basic and advance knowledge of forensic pharmacy because they newly graduated with enough education from the college of pharmacy. The female responders had lower knowledge than females that might relate to their practice or trained at forensic pharmacy services because females are fairer than males in this field and can admit the criminal cases. The pharmacy intern had lower knowledge of forensic pharmacy, and this is normal because they still have not fully finished their forensic pharmacy knowledge and need more education and training in and more practice with future manner. The lower years of experience had lower basic and advance knowledge of forensic pharmacy as expected. The responders are still young and need more time for practice and additional training with short courses or long training in residency programs. The study presented multiple regression analysis, and there was an association between factors and basic and advanced knowledge of forensic pharmacy. The only two positive depending factors number of years experiences and negative dependence factors positions. In the basic knowledge forensic pharmacy, each unit of increase of years involvements the basic knowledge increase with $31 \%$, while each unit of positions increases the knowledge decrease by $15 \%$. In the advanced knowledge of forensic pharmacy, each unit of year's experiences increase the advance knowledge increase by $21 \%$, while each unit of position the advanced knowledge reductions by $11 \%$.

Moreover, with each one-unit increase of location, the advanced knowledge reduced by $16 \%$. The location might clarify the areas of forensic pharmacy services provided to the population. Forensic pharmacy desires more education and training in the college of pharmacy, emphasizing forensic pharmacy residency program.

\section{Limitations}

Various limitations were created in the study includes the unequal distribution of responders among locations, site of work, age, position hold, and several years' experience. In addition, the majority of responders were young age with few years of experience with lower positions. It is representative of their insufficient knowledge about forensic pharmacy and related issues. As a result, it isn't easy to compare the study results with the earlier examination that did not exist.

\section{CONCLUSION}

The current electronic survey with the convenient method and tolerable sample size discussed the pharmacist's knowledge of forensic pharmacy services that was poor. Pharmacists know medication-induced addition or narcotics law, while forensic pharmacist job description with unsatisfactory knowledge. The primary and advanced forensic pharmacy knowledge is exaggerated by the number of year's experiences and career positions. The implementation of education and training of forensic pharmacy is mandatory to improve new services in Saudi Arabia.

\section{ACKNOWLEDGEMENT}

None.

\section{CONFLICT OF INTEREST}

The authors declare that there is no conflict of interest.

\section{Funding}

None

\section{Consent for Publications}

Informed consent was obtained from all the participants

\section{Ethical Approval}

This research was exempted from research and ethical committee or an institutional review board (IRB) approval.

https://www.hhs.gov/ohrp/regulations-andpolicy/decision-charts-2018/index.html

\section{ABBREVIATIONS}

KSA: Kingdom of Saudi Arabia; SPSS: Statistical Package of Social Sciences; JASP: Jeffery's Amazing Statistics Program; Strobe: Strengthening the reporting of observational studies in epidemiology statement: guidelines for reporting observational studies; SFDA: Saudi Food and Drug Authority; CBAHI: Saudi
Central Board for Accreditation of Healthcare Institutions.

\section{ORCID ID}

Yousef Ahmed Alomi org/0000-0003-1381-628X

https://orcid.

\section{REFERENCES}

1. Ahmed Alomi $Y$, Jamaan Alghamdi $S$, Abdullah Alattyh R, Shorog E, Alshahran A, Alasmary S, et al. National survey of pharmacy practice at MOH hospitals in Saudi Arabia 2016-2017: pharmacy management and resource. J Pharm Pract. Commun Med. 2018;4(1s):s1-16.

2. Alomi YA, Alghamdi SJ, Alattyh RA, Shorog E Alshahran A, Alasmary S, Alenazi H, Almutairi A. National survey of pharmacy practice at Ministry of Health Hospitals in Saudi Arabia 2016-2017: prescribing and medication management. J Pharm Pr Community Med. 2018;5:S54-9.

3. Alomi YA, Jamaan Alghamdi $S$, Abdullah Alattyh $R$, Shorog E, Alshahran A, Alasmary S, et al. National survey of pharmacy practice at $\mathrm{MOH}$ hospitals in Saudi Arabia 2016-2017: preparation of medications and dispensing. J Pharm Pract. Commun Med. 2018;4(1s):s54-9.

4. Alomi YA, Alghamdi SJ, Alattyh RA. National survey of pharmacy practice at $\mathrm{MOH}$ hospitals in Saudi Arabia. 2016-2017: Clinical Pharmacy Services. J Pharm Pr Community Med. 2018;4(1):1S-8S.

5. Alomi YA, Shorog E, Alshahrani A, Alasmary S, Alenazi $H$, Almutairi A, et al. National survey of pharmacy practice at $\mathrm{MOH}$ hospitals in Saudi Arabia 2016-2017: drug monitoring and patients education. J Pharm Pract. Commun Med. 2018;4(1s):s17-22.

6. AHSP. ASHP statement on pharmaceutical care. Am J Hosp Pharm. 1993;50(50):1720-3

7. American Society of Health System Pharmacists. ASHP guidelines on a standardized method for pharmaceutical care. American Journal of HealthSystem Pharmacy. 1996;53(14):1713-6. doi: 10.1093/ajhp/53.14.1713.

8. Alsharfa A, Albassri $H$, Alonizi $K$, Alothaian $M$, Alreshidi M AT. National Drug Information Center Services through Ministry of Health Hotline Calling Center. YA1, Alomi, AL- Mudaiheem. Adv Pharmacoepidemiol Drug Saf. 2016;937 in Saudi Arabia:5(0):198.

9. AlomiYA, El-Bahnasawi M, Kamran M, Shaweesh T, Alhaj S, Radwan RA. The clinical outcomes of pharmacist interventions at critical care services of private hospital in Riyadh City, Saudi Arabia. PTB Reports. 2019;5(1):16-9. doi: 10.5530/ PTB.2019.5.4.

10. Alomi YA, Aldosori N, Alhadab M, Alotaibi NR, Al-Shubbar N, Al-Enazi ADM, et al. The outcomes of clinical pharmacist consultation visits at Ministry of Health Hospitals in Saudi Arabia: medication safety and pharmacy research. J Pharm Pract. Commun Med. 2017;3(3).

11. Alomi YA, El-Bahnasawi M, Elemam A, Shaweesh T, Antonio EJ. The economic outcomes of pharmacist interventions at critical care services of private hospital in Riyadh City, Saudi Arabia. PTB Reports. 2019;5(3s):S23-34. doi: 10.5530/ PTB.2019.5.34.

12. Alomi1 YA, Almudaiheem HY. Cost efficiency of national drug information center services through Ministry of Health hotline calling center. In: Saudi Arabia. Int J Pharm Heal Sci. Vol. 937; 2019;2(1). p. 17-20.

13. Anderson PD. An overview of forensic pharmacist's practice. J Pharm Pract. 2000;13(3):179-86.

14. Anderson PD. An overview of forensic pharmacist's practice. J Pharm Pract. 2000;13:179-86. 
15. Anderson PD. The broad field of forensic pharmacy. J Pharm Pract. 2012;25(1):7-12. doi: 10.1177/ 0897190011431144 , PMID 22251569.

16. Anderson PD, Naik K, Kinemond C, ImObersteg A. Forensic testing for drugs of abuse. J Pharm Pract. 2000;13:226-35.

17. Anderson PD, Bokor G. Forensic aspects of druginduced violence. J Pharm Pract. 2012:25(1):41-9. doi: 10.1177/0897190011431150, PMID 22215647.

18. Charan J, Biswas T. How to calculate sample size for different study designs in medical research? Indian J Psychol Med. 2013;35(2):121-6. doi: 10.4103/0253-7176.116232, PMID 24049221

19. Pourhoseingholi MA, Vahedi M, Rahimzadeh $M$. Sample size calculation in medical studies. Gastroenterol Hepatol Bed Bench. 2013;6(1):14-7. PMID 24834239.

20. Ezhumalai DG. How Big A Sample Do I Require?. Annals of SBV. 2017;6(1):39-41. doi: 10.5005/ jp-journals-10085-6113.

21. Johnson TP, Wislar JS. Response rates and nonresponse errors in surveys [internet]. JAMA. 2012;307(17):1805-6. doi: 10.1001/ jama.2012.3532, PMID 22550194.

22. von Elm E, Altman DG, Egger M, Pocock SJ, Gøtzsche PC, Vandenbroucke JP. The strengthening the reporting of observational studies in epidemiology (STROBE) statement: guidelines for reporting observational studies. PLOS Med. 2007;4(10):1623-7. doi: 10.1371/journal. pmed.0040296.

23. Von Elm E, Altman DG, Egger M, Pocock SJ, Gøtzsche PC, Vandenbroucke JP. The Strength- ening the Reporting of Observational Studies in Epidemiology (STROBE) statement: guidelines for reporting observational studies [internet]. Vol. 370; 2007. Available from: http://www.thelancet. com. Available from: http://www.plosmedicine. org [cited 8/19/2021].

24. Liao D, Valliant R. Variance inflation factors in the analysis of complex survey data. Surv Methodol. 2012;38(1):53-62.

25. Akinwande MO, Dikko HG, Samson A. Variance Inflation Factor: As a Condition for the Inclusion of Suppressor Variable(s) in Regression Analysis. Open J Stat. 2015;05(07):754-67.

26. Thompson CG, Kim RS, Aloe AM, Becker BJ. Extracting the Variance In flation Factor and Other Multicollinearity Diagnostics from Typical Regression Results. Basic Appl Soc Psych. 2017;39(2):81-90.

27. Anderson PD, O'Donnell JT. Specific forensic applications for pharmacists. J Pharm Pract. 2000;13(3):187-93. doi: 10.1177/089719000001300305

28. Alomi YA, Alghamdi SJ, Alattyh RA. Hospital pharmacist workforce in eleven-year. J Pharm. 20062016 at Ministry of Health in Saudi Arabia. Pract Community. Med. 2018;4(1):S115-20.

29. Alomi YA, Alghamdi SJ, Alattyh RA. Hospital pharmacist workforce analysis in eleven-year. $J$ Pharm. 2006-2016. Pract Community. Med. 2018;4(1):S103-8.

30. Saudi Ministry of Health. MOH statistical report [internet]. Saudi Arabia: Ministry of Health. p. 1-318; 2017. Available from: https://www. moh. gov.sa/Ministry/About/Documents/MOH

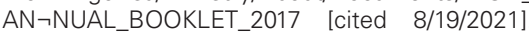
FINAL. Pdf. Vol. 1.

31. Alomi YA, Aldosori N, Alhadab M, Alotaibi NR, Al-Shubbar N, Jadkarim MM, et al. Impact of clinical pharmacist consultation visits at Ministry of Health Hospitals in Saudi Arabia: clinical phar $\neg$ macy services and pharmacy workforce. J Pharm Pract. Commun Med. 2017;3(3).

32. Alomi YA, El-Bahnasawi M, Elemam Al, Elgaili $\mathrm{SH}$, Kamran M. Clinical and economic impact of pharmacist intervention at critical care of private hospital in Riyadh, Saudi Arabia. Value Heal. 2016;7(19):A465.

33. Alomi YA, Aldosori N, Alhadab M, Alotaibi NR Al-Shubbar N, Alotaibi T, et al. The value of clinical pharmacist consultation visits at Ministry of Health Hospitals in Saudi Arabia: intravenous admixture services and pharmacy Total Quality Management. J Pharm Pract. Commun Med. 2017;3(3).

34. Ibrahim IA, Soliman SS, Alzahrani HS. Awareness of medical students toward forensic medicine at Albaha University medical college, Saudi Arabia. J Pak Med Assoc. 2019:69(12):1896-9. doi: 10.5455/ JPMA.263356, PMID 31853124.

35. Abdul NS, Alhazani L, Alruwail R, Aldres S, Asil S Awareness of forensic odontology among undergraduate, graduate, and postgraduate dental students in Riyadh, Saudi Arabia: A knowledge-, attitude-, and practice-based study. J Forensic Dent Sci. 2019;11(1):35-41. doi: 10.4103/jfo.jfds_52_19 PMID 31680754 